\title{
The Exoplanet Microlensing Survey by the Proposed WFIRST Observatory
}

\author{
Richard Barry $^{*}$, Jeffrey Kruk ${ }^{\mathrm{a}}$, Jay Anderson ${ }^{\mathrm{b}}$, Jean-Philippe Beaulieu ${ }^{\mathrm{c}}$, David P. Bennett ${ }^{\mathrm{d}}$, Joseph \\ Catanzarite $^{\mathrm{e}}$, Ed Cheng ${ }^{\mathrm{f}}$, Scott Gaudi ${ }^{\mathrm{g}}$, Neil Gehrels ${ }^{\mathrm{a}}$, Stephen Kane ${ }^{\mathrm{h}}$, Jonathan Lunine ${ }^{\mathrm{i}}$, Takahiro \\ Sumi $^{\mathrm{j}}$, Angelle Tanner ${ }^{\mathrm{k}}$, Wesley Traub ${ }^{\mathrm{e}}$ \\ aNASA, Goddard Space Flight Center, Greenbelt, MD, USA 20771; \\ ${ }^{\mathrm{b}}$ Space Telescope Science Institute, Baltimore, MD USA 20912; \\ 'Institute d'Astrophysique de Paris, Paris, France 75014; \\ ${ }^{\mathrm{d}}$ University of Notre Dame, Notre Dame, IN USA 46556; \\ 'Jet Propulsion Laboratory, Pasadena, CA USA 91109; \\ ${ }^{\mathrm{f}}$ Conceptual Analytics, Glenn Dale, MD USA 20769; \\ g Ohio State University, Columbus OH USA 43210; \\ ${ }^{\mathrm{h}}$ IPAC/California Institute of Technology, Pasadena, CA USA 91125; \\ ${ }^{\mathrm{i}}$ Cornell University, Ithaca, NY USA 14853; \\ ${ }^{\mathrm{j}}$ Osaka University, Osaka, Japan 560-0043; \\ ${ }^{\mathrm{k}}$ Mississippi State University, Starkville, MS, USA 39762.
}

\begin{abstract}
The New Worlds, New Horizons report released by the Astronomy and Astrophysics Decadal Survey Board in 2010 listed the Wide Field Infrared Survey Telescope (WFIRST) as the highest-priority large space mission for the coming decade. This observatory will provide wide-field imaging and slitless spectroscopy at near infrared wavelengths. The scientific goals are to obtain a statistical census of exoplanets using gravitational microlensing, measure the expansion history of and the growth of structure in the Universe by multiple methods, and perform other astronomical surveys to be selected through a guest observer program. A Science Definition Team has been established to assist NASA in the development of a Design Reference Mission that accomplishes this diverse array of science programs with a single observatory. In this paper we present the current WFIRST payload concept and the expected capabilities for planet detection. The observatory, with science goals that are complimentary to the Kepler exoplanet transit mission, is designed to complete the statistical census of planetary systems in the Galaxy, from habitable Earth-mass planets to free floating planets, including analogs to all of the planets in our Solar System except Mercury. The exoplanet microlensing survey will observe for 500 days spanning 5 years. This long temporal baseline will enable the determination of the masses for most detected exoplanets down to 0.1 Earth masses.
\end{abstract}

Keywords: WFIRST, microlensing, exoplanets, dark energy

\section{INTRODUCTION}

The Wide Field Infrared Survey Telescope (WFIRST) mission that is presently under study will provide a powerful tool for obtaining a statistical census of exoplanets that is complementary to that provided by the Kepler mission. In this paper we present the status of the WFIRST project and a summary of the mission design, a description of the microlensing technique, and the expected performance of WFIRST for exoplanet detection.

\footnotetext{
*Richard.K.Barry@nasa.gov; phone 301-286-0664; fax 801-806-7511
}

Techniques and Instrumentation for Detection of Exoplanets V, edited by Stuart Shaklan

Proc. of SPIE Vol. 8151, 81510L · (C) 2011 SPIE · CCC code: 0277-786X/11/\$18 · doi: 10.1117/12.898574 


\subsection{Project History}

The New Worlds, New Horizons report released by the Astronomy and Astrophysics Decadal Survey Board in 2010 listed the Wide Field Infrared Survey Telescope (WFIRST) as the highest-priority large space mission for the coming decade. The scope of this new observatory is intended to encompass a diverse array of science programs: microlensing by exoplanets; characterization of the expansion history of and the growth of structure in the Universe by measurements of type Ia supernovae, weak lensing, and baryon acoustic oscillations; a Galactic plane survey; and a guest investigator program. The recommended baseline for mission concept development was the Joint Dark Energy Mission "Omega" design submitted to Astro-2010. As a first step towards implementation of this project, NASA convened a Science Definition Team in December 2010, with a charter “...to provide science requirements, investigation approaches, key mission parameters, and any other scientific studies needed to support the definition of an optimized space mission concept satisfying the goals of the WFIRST mission as outlined by the Astro2010 Decadal Survey." The interim report of the SDT has recently been released and is available at http://wfirst.gsfc.nasa.gov/science/WFIRST IDRM Report Final signed Rev1.pdf [14]. This interim report is the basis for the present paper; the full study is expected to continue through 2012 and the mission design may well evolve from what is presented here.

\subsection{Microlensing Science Program}

The primary objective of the WFIRST exoplanet science program is to complete the statistical census of planetary systems in the Galaxy, from habitable Earth-mass planets to free floating planets, including analogs to all of the planets

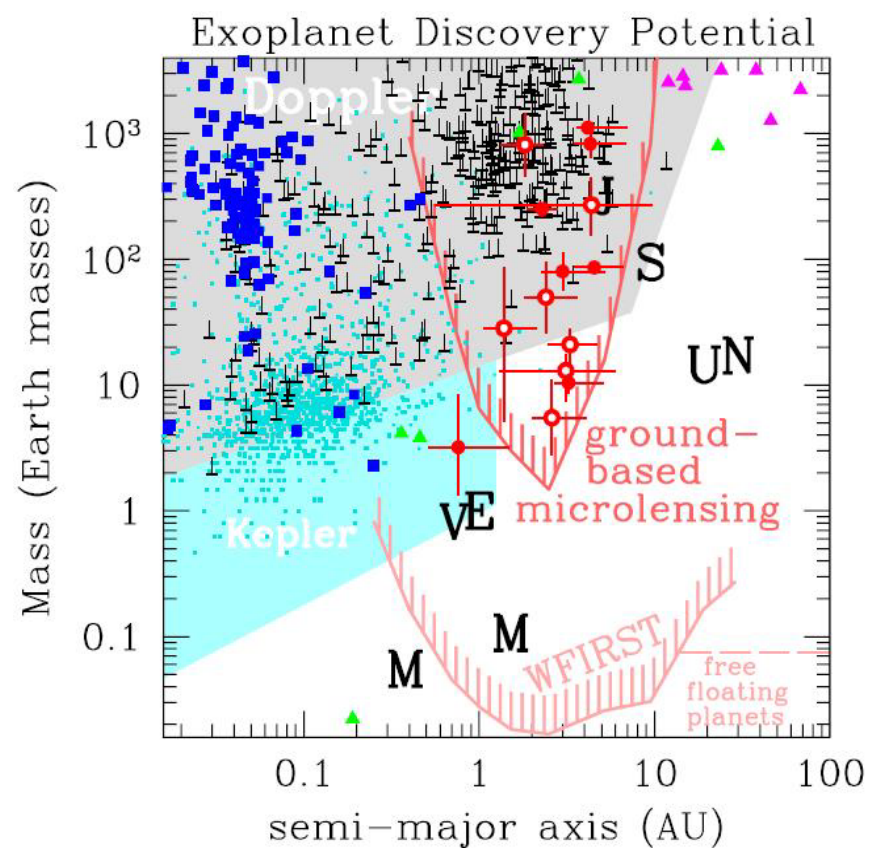

Figure 1 The distribution of known exoplanet masses and semi-major axes is compared to the expected final sensitivity of the Kepler mission and the sensitivity of a 500-day WFIRST Exoplanet survey. Ground based microlensing planets are show as red circles, radial velocity planets are brown X's, planets discovered via their transits are blue squares, and planets found by timing and direct imaging are green and magenta triangles, respectively. The cyan region shows the expected sensitivity of Kepler, and the small darker cyan squares are Kepler's candidate planets. The purple curve shows the sensitivity of a 500-day WFIRST exoplanet program, and the dashed "free-floating planets" line indicates the sensitivity limit for free-floating planets. Some bound planets beyond $10 \mathrm{AU}$ will be discovered without a microlensing signal of their host stars. 
in our Solar System except Mercury. The Kepler planetary mission, which detects planets transiting their host stars, is primarily sensitive to planets with orbital radii below 1 astronomical unit (AU) and radii greater than one Earth radius. To date, Kepler has detected over 1200 candidate planets with a wide array of properties [1]. Using the microlensing technique, WFIRST will probe a region of parameter space complimentary to that of Kepler, as shown in Figure 1. An important aspect of the microlensing technique is the ability to detect free-floating planets. Sumi et al. [2] concluded that Jupiter-mass planets not bound to host stars may be twice as common as stars.

The WFIRST exoplanet science program is driven by two fundamental questions:

1. How do planetary systems form and evolve?

2. How common are potentially habitable worlds?

WFIRST can address the first of these questions by determining the demographics of planetary systems with its sensitivity to virtually all types of exoplanets that will not be detected by Kepler. Furthermore, due to its sensitivity to planets at substantial distances from our solar system, WFIRST can determine how the properties of planetary systems depend on their Galactic environment. WFIRST also has the unique ability to detect free-floating planets down to $\sim 1$ Earth-mass, which provide important constraints on the dynamical histories of planetary systems. Finally, the microlensing technique is also able to detect planets that orbit stars that are too faint to be observed directly. This is an advantage of the technique, but if all the planets detected by WFIRST fall into this category, its contribution to Exoplanet Survey Question \#1 will be significantly compromised. These considerations lead to the following Science Requirements:

A. Make a definitive measurement of the frequency of bound and free-floating planets with masses extending to less than an Earth-mass and separations greater than $0.5 \mathrm{AU}$.

B. Measure the masses of the planets and host stars for the majority of the detected exoplanetary systems.

Kepler will address Exoplanet Survey Question 2, which is critical for the planning of future missions, but this is a challenging measurement for Kepler. So, additional measurements with independent detection methods such as microlensing with WFIRST are needed. This motivates the final WFIRST Science Requirement:

\section{Make a definitive measurement of the frequency of habitable planets.}

\subsection{Planet detection by Microlensing}

WFIRST finds exoplanets using the technique of gravitational microlensing. The physical basis of microlensing is the gravitational distortion of light rays by a star or planet. (See Figure 2.) if a foreground lens star passes near the line of sight to a more distant source star, the gravitational field of the lens star deflects the light rays from the source star resulting in magnification of its image. Consequently the observer sees a microlensing event as a transient brightening of the source as the lens star's proper motion, relative to the source, moves it across the line of sight. In general, the spatial scale of gravitational microlensing events at the lensing star are characterized by the Einstein ring radius,

$$
R_{E}=2.0 \mathrm{AU} \sqrt{\frac{M_{\mathrm{L}}}{0.5 M_{\odot}} \frac{D_{\mathrm{L}}\left(D_{\mathrm{S}}-D_{\mathrm{L}}\right)}{D_{\mathrm{S}}(1 \mathrm{kpc})}},
$$

where $M_{L}$ is the lens mass, and $D_{L}$ and $D_{S}$ are the distances to the lens and source stars, respectively[13]. $R_{E}$ is the radius of the ring image (in the plane of the lens star) that would be seen with perfect alignment between the lens and source stars. A microlensing event's duration is given by the Einstein ring crossing time and is typically 1-3 months for an event produced by a star and a few days or less for an event due to a planet. 


\section{Planetary Microlensing}

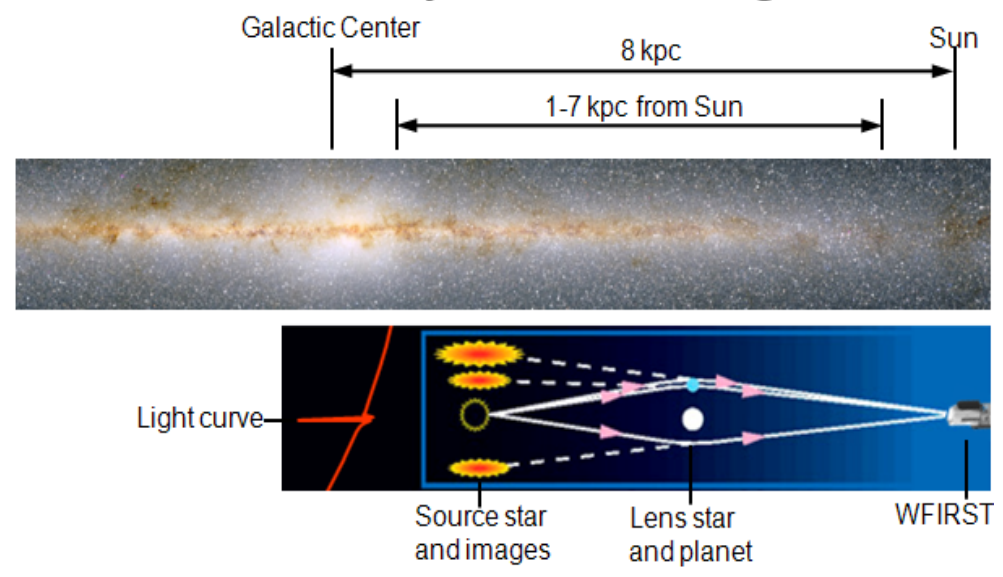

Figure 2. The geometry of a microlensing planet search towards the Galactic bulge. Main sequence stars in the bulge are monitored for brightening due to gravitational lensing by foreground stars and planets in the Galactic disk and bulge.

Planets are detected via light curve deviations that differ from the normal stellar lens light curves [3]. Usually, the planet signal occurs when one of the two images of the source star passes close to the location of the planet, as indicated in Figure 2 (Gould \& Loeb [4]), but planets are also detected at very high magnification where the gravitational field of the planet destroys the symmetry of the Einstein ring (Griest \& Safizadeh [5]). The probability of a detectable planetary signal and its duration both scale as $\mathrm{RE} \sim \mathrm{Mp} 1 / 2$, but with optimum alignment, planetary signals from low-mass planets can be quite strong (many tens of percent). The limiting mass for the microlensing method is reached when the planetary Einstein radius becomes smaller than the radius of the source star (Bennett \& Rhie [6]), resulting in a suppression of the amplitude of the deviation. This suppression is stronger for planets located inside RE. For the F, G or $\mathrm{K}$-dwarf source stars in the bulge that can be monitored with a space-based survey, the sensitivity of the microlensing method extends down to $<0.1$ Earth-mass.

Microlensing is most sensitive to planets at a separation of $\sim R_{E}$ (usually 2-3 AU) due to the strong stellar lens magnification at this separation, but the sensitivity extends to arbitrarily large separations. Planets well inside $\mathrm{R}_{\mathrm{E}}$ have a lower probability of detection. Planets in the habitable zones of their host star tend to be located at separations that are substantially smaller than $\mathrm{R}_{\mathrm{E}}$, and thus are more difficult to detect. This in turn requires the higher photometric precision and higher angular resolution afforded by a space based microlensing mission.

\subsection{Survey Requirements}

Inferring the frequency of exoplanets involves first detecting the planetary deviations, then inferring the planetary parameters from those deviations, and finally calibrating the probability of detecting planets of the given type for the ensemble of observed microlensing events. These pieces of information can then be combined to estimate the intrinsic planet frequency (e.g., Gould et al. [8]). Calibrating the detection probability is a well-understood procedure incurring negligible uncertainties (Gaudi \& Sackett [9]; Rhie et al. [10]), and thus for robustly-measured deviations (as specified below) for which the light curve parameters are well-measured, the fractional uncertainty in the inferred planet frequency is due almost exclusively to the Poisson fluctuations in the number of detected planets $N_{\mathrm{p}}$, which we can 
approximate as $N_{\mathrm{p}}^{-1 / 2}$. Thus achieving a certain precision in the measurement of the exoplanet frequency requires a minimum number of planet detections.

There are a large number of specific questions that we seek to address with the mission products. Fortunately, for most regions of planet discovery space, the number of detections scale self-similarly, such that the ratios of the number of detections in different regions remain approximately constant (Bennett \& Rhie [11]). Thus we can specify the minimum number of detections at one fiducial value (e.g. assuming each star has a 1 Earth-mass planet) to quantify the primary measurement requirement. The resulting set of survey requirements may be summarized as:

1. Planet detection capability to 0.1 Earth Mass. Planets as small as the mass of Mars are thought to be the largest bodies that can be formed by rapid growth of planetary "embryos". Such objects are of particular interest, and can only be detected by microlensing.

2. If every star has a planet with a mass of 1 Earth mass and an orbital period of 2 years, detect (at a $\Delta \chi^{2}>$ 160) at least 120 of them. Here $\Delta \chi^{2}$ is the difference between the $\chi^{2}$ of the model fit with and without a planet, and the minimum value of 160 is sufficient to yield measurements of the planetary mass ratios and projected separations in units of $\mathrm{R}_{\mathrm{E}}$ at precision of $<10 \%$. These detections will facilitate the measurement the mass function of planets with mass $>0.1 \mathrm{M}_{\oplus}$ and separations in the range of 1.5-5 AU with a resolution of 0.2 dex in mass and a precision of $<10 \%$ per mass bin.

3. Measure planet masses to $20 \%$ accuracy for at least 80 planet events with mass of 1 Earth mass and a period of 2 years, assuming one such planet per star. Measuring masses, not just mass ratios, imposes requirements on angular resolution and multi-band imaging for determination of the spectral types of the host stars.

4. If every $F, G$, and $K$ star has a habitable planet, detect (at a $\Delta \chi^{2}>50$ ) at least 25 of them. This is sufficient to enable determination of the frequency of habitable planets, $\eta_{\oplus}$, to a precision of $\sim 20 \% \eta_{\oplus}^{-1 / 2}$. This capability does not follow automatically from the earlier requirements, because the amplitudes of the signals are generally smaller; hence the lower $\Delta \chi^{2}$ threshold.

5. If there is one free floating Earth-mass planet per star in the Galaxy, detect (at a $\left.\Delta \chi^{2}>300\right)$ at least 30 of them. The detection of free-floating planets requires a higher $\Delta \chi^{2}$ threshold than is needed for planets detected with a host star because $\sim 10^{8}$ light curves must be searched for free-floating planetary events, whereas we expect only a few $\times 10^{4}$ stellar microlensing events to be searched for the signals of bound planets.

\section{WFIRST OBSERVATORY}

The WFIRST observatory will be placed in a halo orbit near the second Sun-Earth Lagrange point. The spacecraft bus will be based on that of the Solar Dynamics Observatory (SDO), augmented to provide the fine-pointing control required for WFIRST operations. A schematic view of the observatory is shown in Figure 3. The fixed solar array is mounted on the Sun-shield, and is sized to provide power with suitable margins for telescope pointing at any position in the Field of Regard (shown in Figure 4), while maintaining a fixed S/C roll orientation during the microlensing observing campaigns. The boresight-to-Sun angle can range from $54^{\circ}$ to $126^{\circ}$, allowing 72 -day continuous monitoring of the Galactic bulge. The baseline pointing performance requirements are: 40 milli-arcsec jitter, 25 mas revisit accuracy, 15 mas relative accuracy for dithers. Slew and settle times range from $16 \mathrm{sec}$ for short dithers, up to $38 \mathrm{sec}$ for the $0.7^{\circ}$ degree slews typical of survey operations. The telescope and instruments are passively cooled to minimize thermal emission in the instrument bandpass and detector dark current. The instrument focal plane assembly (FPA) radiators are located on the anti-Sun side of the observatory, as shown in Figure 3. A hydrazine propulsion system handles orbit insertion, station-keeping, and momentum management.

The science payload consists of a $1.3 \mathrm{~m}$ unobscured focal three-mirror anastigmat (TMA) telescope feeding three instrument channels: an imaging channel (ImC), and two spectrometer channels (SpC). The field of view (FOV) of each instrument channel is defined by a pickoff mirror located at the intermediate focus formed by the primary and secondary mirrors. Each instrument channel thus has a dedicated tertiary mirror. The only optics following the tertiary in the ImC 
are fold mirrors and the filter wheel; the SpCs include a dispersing prism and a lens assembly to adjust the focal length. The field of view of the instrument channels as projected onto the sky is shown in Figure 4. The ImC is well-corrected over the entire $0.463^{\circ} \mathrm{x} 0.802^{\circ} \mathrm{FOV}$ : design residuals give significant margin for diffraction-limited performance over the full field and over the full bandpass. The SpC channels are not used for the microlensing program and are not discussed further here. The optical design and its performance are described in detail by Content et al. [12].

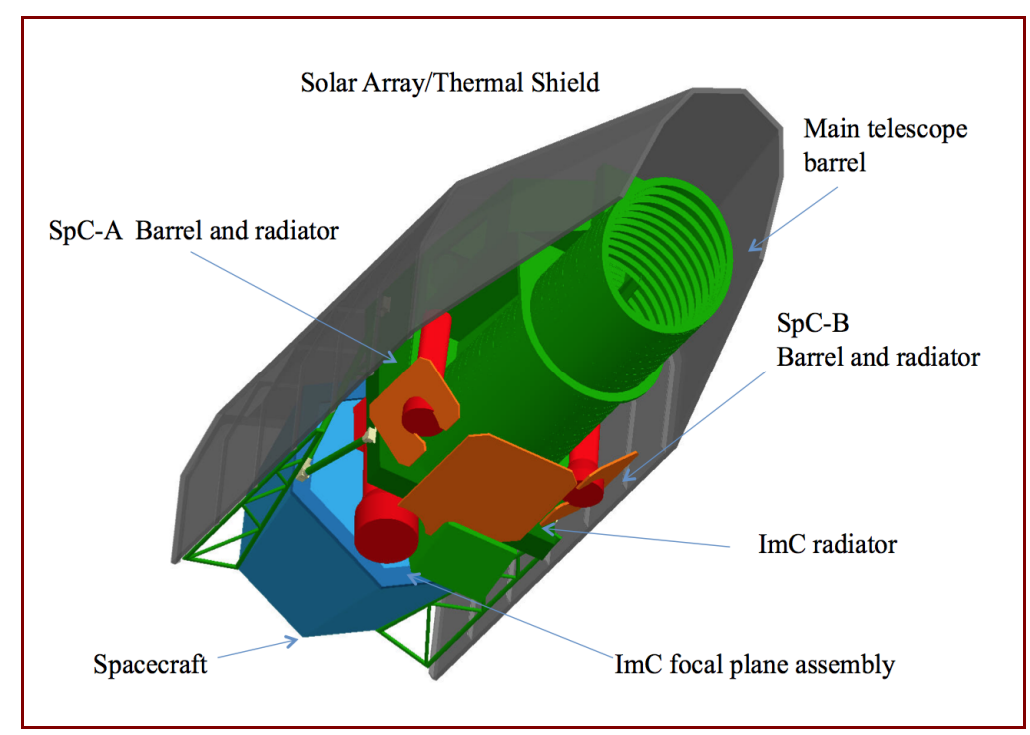

Figure 3. Schematic view of the WFIRST observatory.

The ImC contains a 7-position filter wheel, located at a cold pupil. The filter complement is given in Table 1. Most observations in the microlensing program will be performed with the W149 filter to maximize the detected signal. At regular intervals, each field in the exoplanet survey will also be observed with the F087 filter to obtain colors of the lensed stars for determining spectral types. Observations with one or more of the other filters may also be obtained to further constrain the lens star properties.

The FOV of the WFIRST instrument is shown in Figure 4, as projected onto the sky. The full FOVs of HST and JWST are shown for comparison. Each square in the WFIRST focal plane is a $2 \mathrm{Kx} 2 \mathrm{~K}$ HgCdTe sensor chip array (SCA). The $\operatorname{ImC}$ is a $7 \mathrm{x} 4$ array of SCAs, with a plate scale of $0.18 \% /$ pixel. The SpC plate scale is $0.45 \% /$ pixel. The ImC focal plane contains four "outrigger" SCAs in addition to the $7 \times 4$ array of science SCAs. These outrigger detectors will be used to provide a fine-guiding signal for the attitude control system. Only two are needed for this purpose, with the other two provided for redundancy.

Table 1. WFIRST filter wheel usage. 'DE' indicates the dark energy program.

\begin{tabular}{|c|c|c|}
\hline Position/Name & Bandpass $(\boldsymbol{\mu m})$ & Usage \\
\hline Filter, F087 & $0.76-0.97$ & Exoplanets, DE, surveys \\
\hline Filter, F111 & $0.97-1.24$ & DE, surveys \\
\hline Filter, F141 & $1.24-1.57$ & DE, surveys \\
\hline Filter, F178 & $1.57-2.00$ & DE, surveys \\
\hline Filter, W149 & $0.97-2.00$ & Exoplanets \\
\hline Prism, P130 & $0.60-2.00$ & DE \\
\hline Blank & N/A & Calibration \\
\hline
\end{tabular}




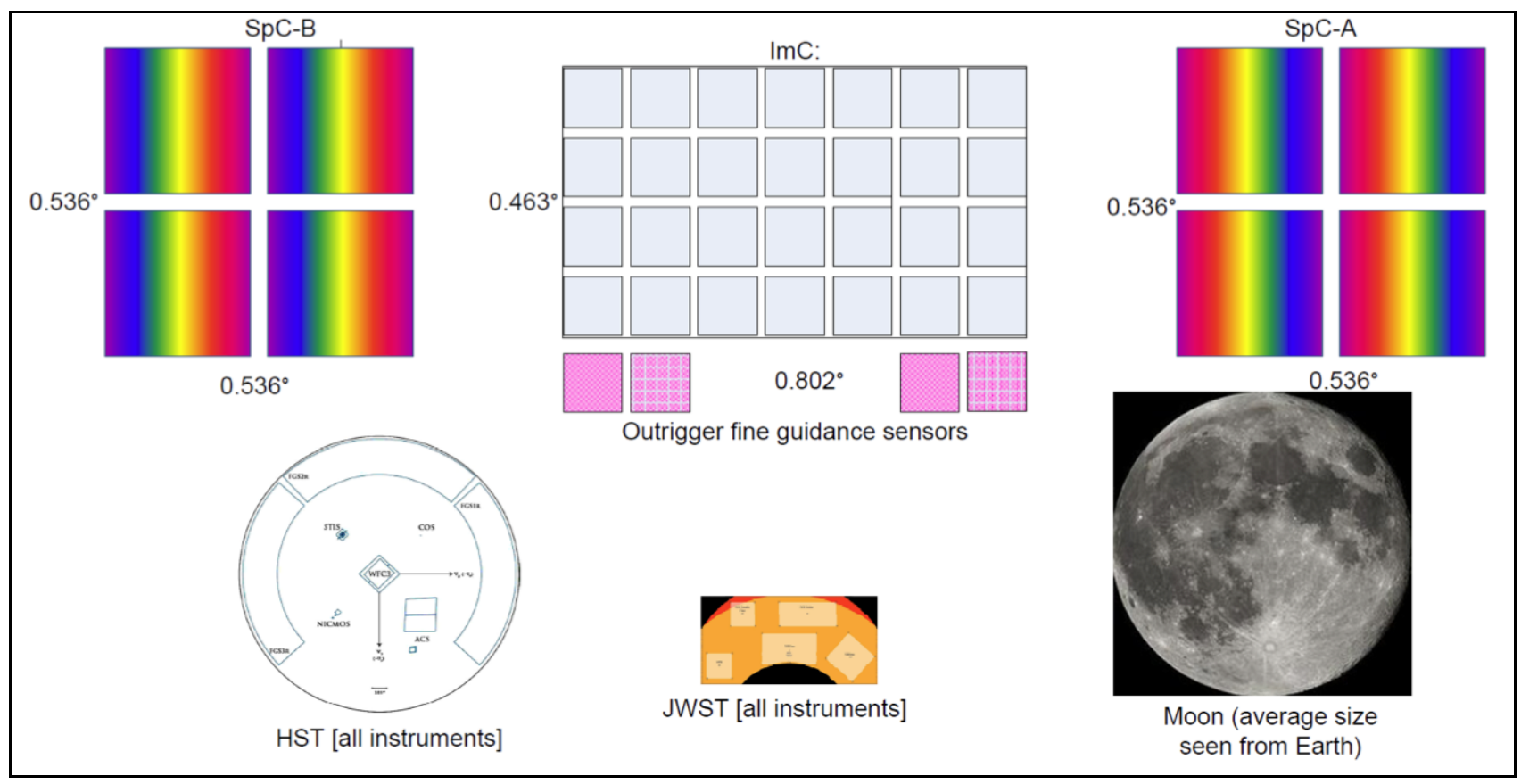

Figure 4. The FOV of the WFIRST instrument is shown, as projected onto the sky. The full FOVs of HST and JWST are shown for comparison. Each square in the WFIRST focal plane is a $2 \mathrm{~K} \times 2 \mathrm{~K} \mathrm{HgCdTe}$ sensor chip array (SCA). The ImC is a $7 \times 4$ array of SCAs, with a plate scale of $0.18 \% /$ pixel. The SpC plate scale is $0.45 \% /$ pixel.

\section{PREDICTED PERFORMANCE}

\subsection{Baseline microlensing observing program.}

Microlensing requires precise alignment of the foreground and background stars, hence such events are rare even for high-density lines of sight. The probability of a detectable perturbation of the stellar microlensing event by a planet orbiting the foreground star ranges from as high as several tens of percent for Jupiter-mass planets to under one percent for planets under one Earth mass. The magnitude of typical perturbations varies similarly: from tens of percent for highmass planets to a few percent for the lowest-mass planets. The observed distribution of event time scales (Figure 7) indicates that the time scale of the primary stellar lensing event is approximately 40 days[13]. The duration of a planetary perturbation is much shorter: a few days for a Jupiter-mass planet to a few hours for an Earth-mass planet. The interval of time between planetary events and the peak of the corresponding stellar events are a priory unknowable. Consequently, monitoring of any given field must be performed continuously with a high sampling cadence.

In order to maximize the number of microlensing events, WFIRST will observe a set of adjacent fields in the Galactic bulge. Observing in the near-infrared (NIR) significantly reduces the effects of extinction due to galactic dust, increasing the number and apparent brightness of the background stars. The exposure time will be 88 seconds per field, with a slew and settle time of 38 seconds between fields. One pass through the seven fields requires 15 minutes, after which the sequence is repeated. This cadence provides good sampling of the shortest planetary microlensing light curves. Most observations will be performed with the W149 filter, which spans $0.97 \mu \mathrm{m}-2.0 \mu \mathrm{m}$, to maximize the signal-to-noise of microlensing events (which are achromatic). Observations with the F087 filter will be obtained once every 12 hours. This sequence will be repeated throughout a 72-day bulge observing campaign, interrupted only for momentum dumping and other occasional events such as calibration and acquisition of data from targets of opportunity. It is anticipated that seven such campaigns will be distributed over the five-year mission duration. This is done for the purpose of 
maximizing the proper-motion displacement between the first and last campaigns of the source stars relative to the corresponding lens stars, which will allow us to disentangle the properties of the source/lens stellar pairs.

\subsection{Exoplanet Survey Simulation and Lightcurve examples}

Because the source star density and event rate are strong functions of Galactic coordinates, and the detection probability of a planet with a given set of properties depends sensitively on the detailed properties of the event (host star mass and distance, event duration, source star brightness and angular size), quantitative predictions for the yields of a given realization of exoplanet survey dataset require sophisticated simulations that incorporate models for the Galactic distribution of lenses and sources to simulate and evaluate the detectability of events with realistic photometric precision. Such simulations for the WFIRST exoplanet observing program have been performed, updated from the simulations of Bennett \& Rhie (2002)[11], in order to predict the exoplanet detection sensitivity of the mission.
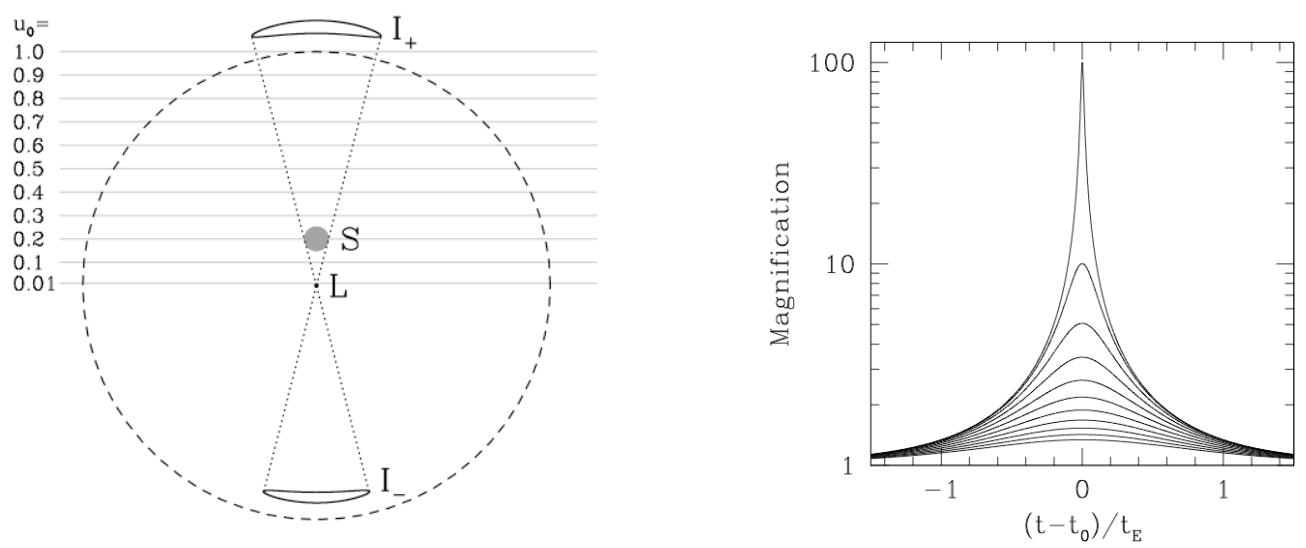

Figure 5. The left panel shows two images $I_{+}$and $I_{-}$created when a source star, $S$, passes nearly but not quite behind a lens star, $\mathrm{L}$. The dimensionless impact parameter $\mathrm{u}_{\mathrm{o}}$ is the smallest angular distance between the source and the lens, measured in units of the Einstein radius. The images shown are for the case in which $u_{o}$ is 0.2 . The right hand panel shows light curves corresponding to the values of $u_{o}$ shown in the left panel, with the change in brightness in stellar magnitude and the time in units Einstein ring crossing time. From top to bottom $\mathrm{u}_{\mathrm{o}}=0.01,0.1,0.2,0.3,0.4,0.5,0.6,0.7,0.8,0.9,1.0$. From [13]. If the source star were to pass precisely behind the lens star $\left(\mathrm{u}_{\mathrm{o}}=0\right)$, the image of the source star would form a perfect 'Einstein Ring', represented by the dashed line in the left panel. This corresponds to maximum magnification and, thus, maximum brightening of the source.

A microlensing light curve in the simplest case of a single star lensing a background star possesses a symmetric form in which the magnification of the source star by the foreground lens star increases as the alignment between the source and the lens becomes more perfect (figure 5). In the presence of a planet around the lens star, near the Einstein ring, the resulting light curve of the source star is distorted in different ways, depending on the position of the planet relative to its host star's Einstein ring, the planet-to-star mass ratio, the possible presence of multiple planets, and even the presence of a moon. Shown in figure 6 are several example lightcurves for different cases of planets around host stars, generated by Bennett and Rhie (2002) [11]. 


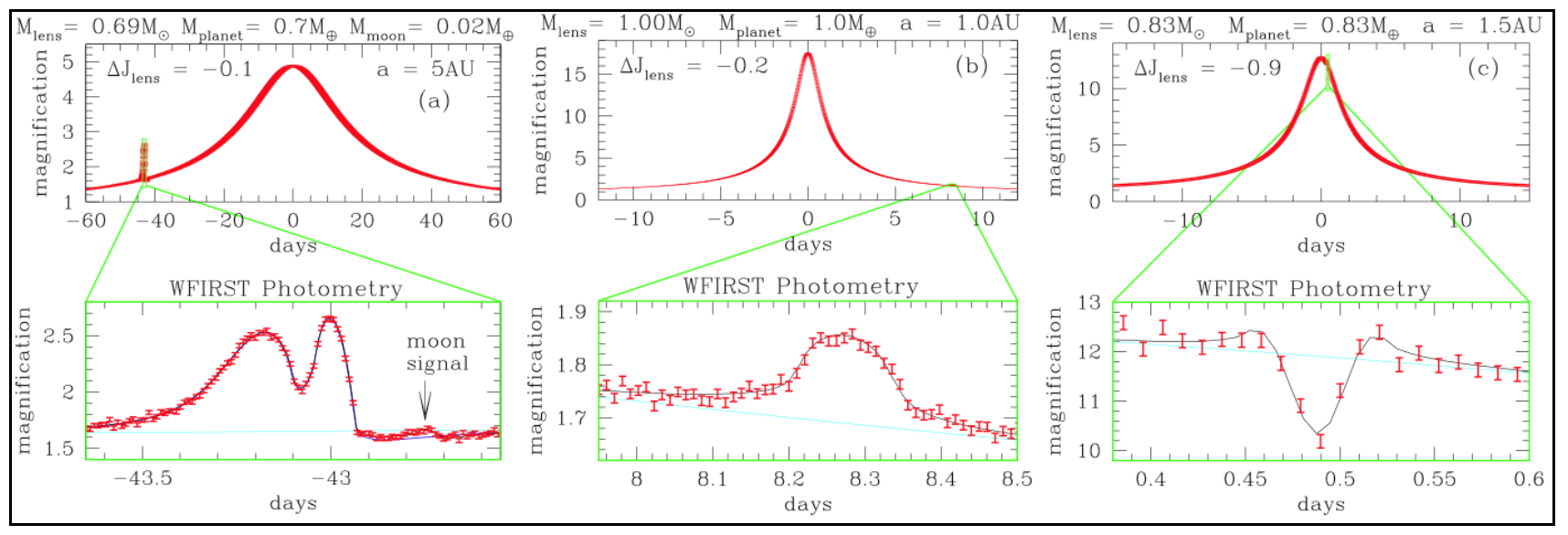

Figure 6. Simulated exoplanet microlens light curves for the WFIRST observatory, based on the simulations of Bennett \& Rhie (2002) [11], which have been updated to reflect the properties of the WFIRST exoplanet survey. All three light curves in the lower row are perturbations due to planets of an Earth-mass or less of a microlens event in the first row and as described in Figure 5. Light curve (a) is for an Earth-mass planet in a wide orbit with a moon of 1.3 lunar masses, and light curves (b) and (c) show the signals of planets close to an Earth-mass in or near the habitable zone. In all three examples, the exoplanet host star is brighter than the source star, which enables a precise determination of the masses of the host star and its planet [16]. $\Delta \mathrm{J}_{\text {lens }}$ is the J-band apparent magnitude difference between the source and lens star.

\subsection{Free-floating planets}

The discovery of hot Jupiters, as well as evidence for the migration of the giant planets in our own solar system, have highlighted the fact that planet formation theories must account for the possibility of large-scale rearrangement of planet positions through gravitational and gas dynamical effects during and after the epoch of planet formation. Many of these theories also predict a substantial population of "free-floating" planets ejected from their planetary systems through gravitational interactions with other planets. Evidence for such a new population of Jupiter-mass planets loosely bound or unbound to any host star was recently found using microlensing[2]. This study found ten single-lens microlensing events with Einstein Radius crossing timescale, $t_{\mathrm{E}}$, of less than 2 days that are most likely due to the solitary Jupiter-mass lenses. Although the lens mass, distance and source-lens relative velocity are degenerate in $t_{\mathrm{E}}$, the lens mass distribution as represented in $t_{\mathrm{E}}$ can be statistically derived using a relatively well known galactic model. (See Figure 7.) This work concluded that "free-floating" planets may outnumber the stars in our galaxy two to one. The interpretation of the final exoplanetary system architectures that we observe today must account for these dynamical processes.

WFIRST can detect "free-floating" planets below an Earth-mass, a task not possible from the ground. Exoplanet microlensing from the ground has had well-documented successes, but just as is the case for transits, space is a vastly better place to carry out statistically significant observations. Current ground-based observations are sensitive only down to Jupiter-mass free-floating planets, and even the next generation ground-based observations cannot detect Earth-mass objects in the next decade. Space-based microlensing observations are superior because of the possibility of continuous observation for weeks at a time with low systematic effects such as variable seeing. This is critical for the very short (a few days to hours) timescale of planetary-mass microlensing events. Furthermore, the stable, high angular resolution images over wide fields of view afforded by a space-based microlensing observatory will enable the detection of host star effects - a task that is impossible from the ground. WFIRST will detect 2080 free-floating exoplanets, including 190 sub-Earth-mass free-floating planets and 480 sub-ten-Earth-mass free-floating planets, assuming the abundance extrapolated from the ground-based measurement[2]. We also expect $\geq 30$ free-floating planets of 1 Earth-mass in a 500day survey assuming one such planet per star. The large number of detections spanning a wide range of masses will be sufficient to address the question of whether the ejection of planets is a phenomenon associated only with giant planet formation, or also involves terrestrial planets. 


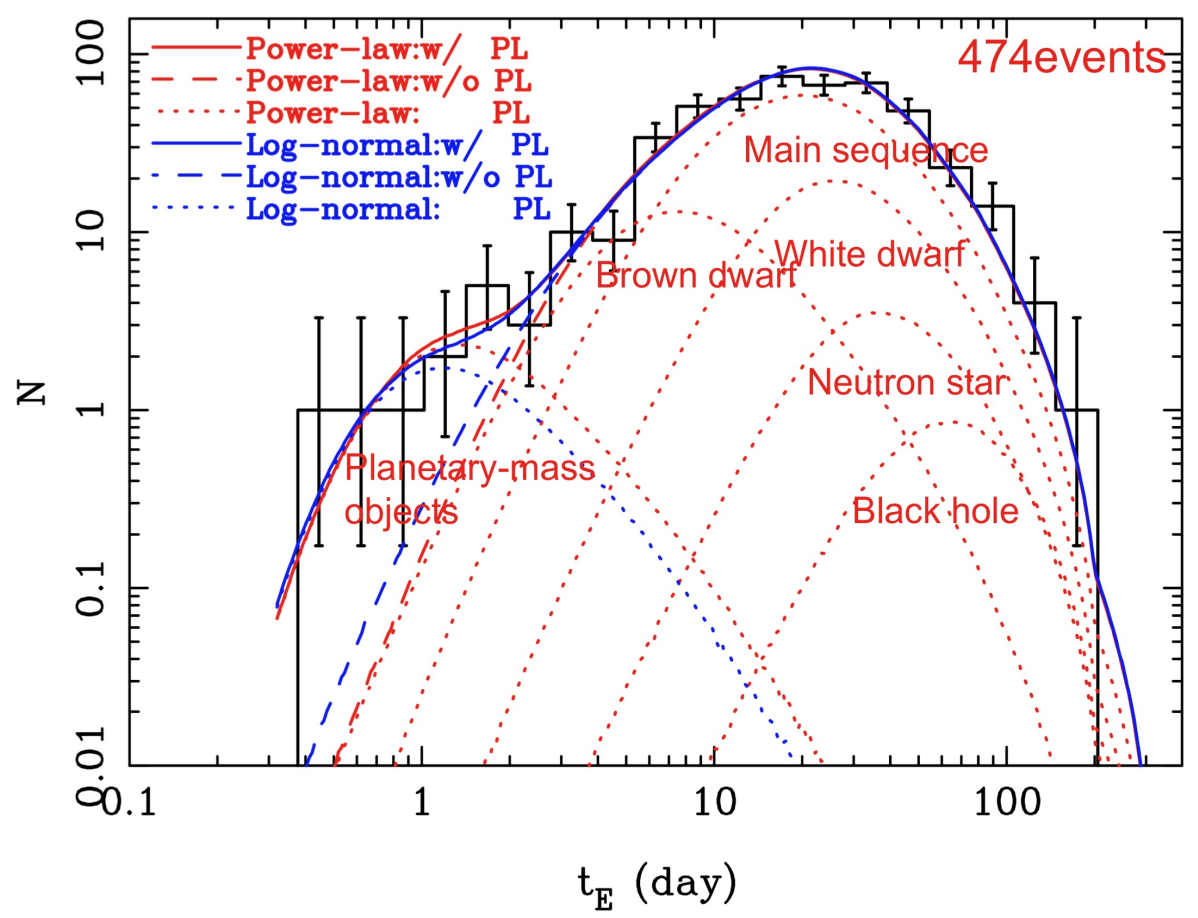

Figure 7. Ground-based observed and theoretical distributions of the event timescale, $\mathrm{t}_{\mathrm{E}}$ (black histogram). The red and blue lines indicate the best-fit models with (1) the power-law and (2) log-normal mass functions, respectively. For both mass functions, Sumi, et al., assumed that stars that were initially above $1 \mathrm{M}_{\text {Sun }}$ have evolved into stellar remnants - white dwarfs, neutron stars or black holes depending on the initial mass. Each model is multiplied by the detection efficiencies. In each model, dashed lines indicate models of the sum of stellar, stellar remnant and brown dwarf populations, and the dotted lines represent the planetary-mass population (PL). The individual populations for the power-law model are also shown by the red dotted lines. Solid lines are the sums of all populations, and both models fit the data well. WFIRST will explore the region down to $t_{\mathrm{E}} \sim 0.01$ day corresponding Mars-mass with large-number statistics so that the mass function of freefloating planet can be measured.

\subsection{Uncertainties}

Owing to the continuous coverage, excellent photometric precision, low systematic errors, and high threshold for detection $\left(\Delta \chi^{2}>160\right)$, the majority of the planetary perturbations found by WFIRST (such as those shown in Figure 6) will be well sampled temporally and thus well characterized. Such light curves yield unambiguous planet mass ratios and separations in units of $R_{E}$, with precisions of $<10 \%$. Planet masses and separations in physical units are needed to place the microlensing detections in the context of the discoveries made by other methods. Converting from mass ratios and projected separations in units of $\mathrm{R}_{\mathrm{E}}$ to planet masses and separations in physical units requires measuring the masses and distances of the host stars. Measuring the mass and distance to a host star in turn requires additional information such as detection of its total flux and color, which will only be obtainable for a subset of the detections. For a small fraction of planetary microlensing events, the lens mass and distance can be measured from second-order effects in the microlensing light curves alone. However, for most of the events, light from the host star can be isolated and measured. This in turn requires high-resolution $\left(<0.3^{\prime \prime}\right)$ imaging in order to resolve out the dense stellar background in the bulge fields, and so allow for the unambiguous identification of the light from the host. Together with information routinely measured from the light curve, a measurement of the host light enables the determination of its mass and distance, and so planet masses and separation in physical units (Bennett et al. 2007). Observations spread over the course of the 5-year mission will also enable separate determination of the host lens and source star brightnesses and colors as they move apart due to their relative proper motion. This can provide redundant information that can be used to confirm the inferred host star parameters. 
Based on extending the results from simulations of the Microlensing Planet Finder (MPF) mission[11], WFIRST should be able to uniquely identify the light from host stars and so measure host star masses to $20 \%$ accuracy for the majority of the planet detections. The detection threshold for habitable planets $\left(\Delta \chi^{2}>50\right)$ is set lower than that for the majority of the planetary perturbations $\left(\Delta \chi^{2}>160\right)$. Thus, the planetary parameters will have correspondingly larger uncertainties, and in particular there may be a degeneracy between the inferred mass ratio and angular size of the source. However, habitable planets will only be found around relatively bright host stars, and thus the lens-source relative proper motion can be detected directly from the images using the method describe above. This enables an estimation of the angular source size, thus breaking the degeneracy with the mass ratio in such low signal-to-noise ratio planetary deviations. Thus the majority of the habitable planets detected by WFIRST should also be well-characterized, despite their lower signal-to-noise ratios.

\subsection{Event yields}

As described above in Section 3.2, we have developed a simulation of the WFIRST exoplanet program[14], based on the earlier simulations of Bennett \& Rhie (2002)[11]. But, in order to estimate the exoplanet detection yields, we must make some assumptions regarding the prevalence of exoplanets. Previous microlensing results, $[2,8,15]$ are the best basis for extrapolation because they sample the same set of exoplanet host stars that will be probed by WFIRST. Simple extrapolations of the bound [8,15] and free-floating [2] exoplanet populations have been used to estimate the WFIRST exoplanet detection yields. The predicted event yields are:

- 3250 total bound exoplanets in the range 0.1-1000 Earth masses and separations in the range 0.1-40 AU, including 320 sub-Earth-mass planets and 1500 sub-ten-Earth-mass planets.

- 2080 free-floating exoplanets, including 190 sub-Earth-mass and 480 sub-ten-Earth-mass free-floating planets.

- If each star hosts planets with the masses and semi-major axes as those in our Solar system, WFIRST will detect 280 terrestrial planets (Venus/Earth/Mars analogs), 3200 gas giants (Jupiter/Saturn analogs), and 84 ice giants (Neptune/Uranus analogs).

\section{SUMMARY}

The WFIRST observatory was recommended by the Astronomy and Astrophysics Decadal Survey Board in 2010 as the highest-priority large space mission for the coming decade. The observatory, which is complimentary to the Kepler exoplanet transit mission already on orbit, will complete the statistical census of exoplanets using the technique of gravitational microlensing. WFIRST is capable of determining the masses of exoplanets from 0.1 to 1000 Earth masses including planets in the habitable zones about main sequence stars. The instrument is most sensitive to planets at orbital separations not sampled by Kepler and will provide data crucial for testing planetary system formation theories. WFIRST will also provide the only means of detecting recently discovered free-floating planets down to an Earth mass. In addition, this observatory will be used to measure cosmic acceleration by multiple methods, and perform other astronomical surveys to be selected through a guest observer program. The WFIRST Science Definition Team established to assist NASA in the development of a Design Reference Mission together with the scientists and engineers of the NASA WFIRST project team have confirmed the basic plausibility of using a single spaceborne observatory to accomplish this diverse array of science programs. 


\section{REFERENCES}

[1] Borucki et al., "Characteristics of Planetary Candidates Observed by Kepler, II: Analysis of the First Four Months of Data", ApJ, 736, 19 (2011).

[2] Sumi, T., et al., "Unbound or Distant Planetary Mass Population Detected by Gravitational Microlensing", Nature, 473, 349 (2011).

[3] Mao, S., \& Paczynski, B., "Gravitational Microlensing by Double Stars and Planetary Systems”, ApJ, 374 , L37 (1991).

[4] Gould, A., \& Loeb, A., "Discovering Planetary Systems Through Gravitational Microlenses", ApJ, 396, 104 (1992).

[5] Griest, K., \& Safizadeh, N., "The Use of High-Magnification Microlensing Events in Discovering Extrasolar Planets", ApJ, 500, 37 (1998).

[6] Bennett, D.P., \& Rhie, S.H., "Detecting Earth-Mass Planets with Gravitational Microlensing", ApJ, 472, 660 (1996).

[7] Bennett, D.P., et al., "Characterization of Gravitational Microlensing Planetary Host Stars”, ApJ, 660, 781 (2007).

[8] Gould et al., "Frequency of Solar-like Systems and of Ice and Gas Giants Beyond the Snow Line from Highmagnification Microlensing Events in 2005-2008", ApJ, 720, 1073 (2010)

[9] Gaudi, B.S., \& Sackett, P.D., "Detection Efficiencies of Microlensing Data Sets to Stellar and Planetary Companions", ApJ, 528, 56 (2000)

[10] Rhie, S., et al., "On Planetary Companions to the MACHO 98-BLG-35 Microlens Star", ApJ, 533, 378 (2000)

[11] Bennett, D.P., \& Rhie, S.H., "Simulation of a Space-based Microlensing Survey for Terrestrial Extrasolar Planets", ApJ, 574, 985 (2002).

[12] Content, D.A., et al., "Optical design trade study for the Wide Field Infrared Space Telescope [WFIRST]", Proc. SPIE 8146 (2011) in press.

[13] Gaudi, B.S., "Exoplanetary Microlensing", in Exoplanets, ed. Sara Seager, University of Arizona Press (2009)

[14] Green, J., et al., "Wide-Field InfraRed Survey Telescope (WFIRST) Interim Report”, (arXiv:1108.1374) (2011).

[15] Sumi, T., et al., “A Cold Neptune-Mass Planet OGLE-2007-BLG-368Lb: Cold Neptunes Are Common”, ApJ, 710, $1641(2010)$.

[16] Bennett, D.P., Anderson, J., \& Gaudi, B.S., "Characterization of Gravitational Microlensing Planetary Host Stars", ApJ, 660, 781 (2007) 